\title{
La dimension symbolique du repas dans la dramaturgie néo-hellénique
}

Le dîner en tant que seuil dans les œuvres théâtrales de Pavlos Matessis et de Dimitris Dimitriadis

The Symbolic Dimension of the Dinner in New Greek Dramaturgy: Eating as a Threshold in Plays of Pavlos Matesis and Dimitris Dimitriadis

\section{Thalia Bousiopoulou}

\section{OpenEdition} Journals

Édition électronique

URL : https://journals.openedition.org/ceb/6350

DOI : $10.4000 /$ ceb.6350

ISSN : 2261-4184

Éditeur

INALCO

Édition imprimée

ISBN : 978-2-85831-230-6

ISSN : 0290-7402

Référence électronique

Thalia Bousiopoulou, «La dimension symbolique du repas dans la dramaturgie néo-hellénique », Cahiers balkaniques [En ligne], Hors-série | 2016, mis en ligne le 14 mars 2017, consulté le 06 juillet 2021. URL : http://journals.openedition.org/ceb/6350; DOI : https://doi.org/10.4000/ceb.6350

Ce document a été généré automatiquement le 6 juillet 2021.

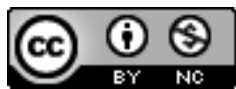

Cahiers balkaniques est mis à disposition selon les termes de la Licence Creative Commons Attribution - Pas d'Utilisation Commerciale 4.0 International. 


\section{La dimension symbolique du repas dans la dramaturgie néo-hellénique}

Le dîner en tant que seuil dans les œuvres théâtrales de Pavlos Matessis et de Dimitris Dimitriadis

The Symbolic Dimension of the Dinner in New Greek Dramaturgy: Eating as a Threshold in Plays of Pavlos Matesis and Dimitris Dimitriadis

Thalia Bousiopoulou

\section{La formation du seuil : manger au théâtre}

1 Manger constitue, d'un certain point de vue, une rencontre. Tant l'acte lui-même que les circonstances qui l'entourent dessinent parfois un champ de l'intermédiaire, donc $\mathrm{du}$ liminaire, où se rencontrent des éléments hétérogènes. En tant qu'acte, la consommation de nourriture met le corps en contact avec le différent, l'Autre pénètre le familier. En tant que circonstance, le rassemblement de convives autour de la table du repas est souvent lié dans l'histoire des communautés humaines à un discours symbolique : les expériences vécues et les toasts-souhaits échangés lors de ces repas pris en commun soulignent la fonction performative de la langue qui n'est plus alors entendue comme énonciation langagière équivalente à un acte, mais dans sa double conception du rôle de la langue : comme organe du corps en même temps que discoursparole. L'art dramatique, d'un autre côté, constitue peut-être le champ par excellence de l'intermédiaire. Sur la scène du théâtre, comme lors de la consommation de nourriture, le familier rencontre l'étranger, le corps de l'acteur rencontre le rôle qu'il incarne, tandis que le spectateur participe à la fois à la fiction scénique et au monde réel auquel appartiennent tant les acteurs que lui-même. Pourtant, à l'intérieur du texte dramatique surgissent parfois aussi des instants au cours desquels s'interrompt le cours continu de l'action, et où l'identité rencontre l'altérité: ce faisant, les personnages demeurent en suspens. Cette zone de transposition constante des références stables peut être qualifiée de seuil. Dans les œuvres théâtrales que nous allons aborder par la suite, le repas constitue un de ces seuils puisque d'un côté, son 
caractère festif-commémoratif permet dans ces circonstances la formation d'un champ sémantique distinct à l'intérieur du texte dramatique, et que de l'autre, par la médiation de la nourriture ou de l'acte de manger, le repas met les personnages dramatiques en opposition avec la dimension matérielle du discours, avec son incarnation.

2 On définit généralement comme seuil le point de passage à un lieu et à un temps différents, mais il constitue aussi l'expérience de ce passage, qui est celle de la métamorphose (STAVRIDIS, janvier-février 1999, 112). Cette notion présuppose des pratiques au symbolisme fort, qui va du lieu de rencontre du dipôle masculin-féminin des habitations kabyles, ainsi que les a abordées Pierre Bourdieu (2000), aux passages du flâneur parisien de Walter Benjamin (2000, 44-46). Puisque le seuil appartient simultanément à deux mondes, celui de l'avant et de l'après, du dedans et du dehors, il permet la coexistence et l'influence réciproque de systèmes symboliques qui se trouvent à la fois dans une relation d'opposition et de similarité (STAVRIDIS, janvierfévrier 1999, 111). Les éléments différents qui entrent en contact dans cette zone ne fusionnent pas, mais ne dressent pas non plus de barrières infranchissables entre eux cette coexistence d'un type particulier conduit souvent à leur ouverture vers de nouveaux champs sémantiques. "La rencontre sur le seuil ", note Stavros Stavridis, « est une rencontre qui finalement prouve ou démontre l'existence de ces endroits où les identités, les totalités, les entités demeurent ouvertes » $(1998,60)$. Tout comme le gestus distanciateur du théâtre brechtien, le seuil interrompt le continuum spatiotemporel afin de marquer la possibilité de déviation et, probablement aussi, d'annoncer la naissance imminente de cette déviation (STAVRIDIS, avril-septembre 1999, 94-98). Dans les deux pièces que nous allons examiner, le signe de cette déviation est différent. Il est positif dans le cas de Bruit ${ }^{1}$ de Pavlos Matessis, dans la mesure où les reliefs du festin de Thyeste vont constituer la voie de renaissance du mythe. Négatif, dans le cas de la pièce le Vertige des animaux avant l'abattage ${ }^{2}$ de Dimitris Dimitriadis, puisque la rencontre ou le repas de la famille va signifier le début de sa destruction par la fonction subitement dangereuse de la langue. Un trait commun aux deux cas est le rôle fondamental du repas qui ouvre à l'altérité les identités des personnages dramatiques.

\section{Le Bruit de Pavlos Matessis}

3 L'intrigue de Bruit de P. Matessis se déroule dans le palais de Mycènes transformé en musée. Là, dans un état se situant entre la vie et la mort, dans une singulière condition de fossilisation, vivent Clytemnestre, Électre et Égisthe. Au centre de la scène se trouvent les reliefs du festin de Thyeste, en souvenir duquel les héros accomplissent chaque année une sorte de cérémonie commémorative lors de laquelle leur est concédé le droit au rêve ou celui de pénétrer dans les rêves des autres. Un groupe de touristes visite le palais sous la conduite d'une guide; parmi eux se trouve Oreste, qui va revenir tuer Clytemnestre. Par la suite, il sera assassiné par Électre.

4 Il est évident que P. Matessis prend pour point de départ la constatation de la standardisation dans le présent du mythe grec antique, de la transformation des personnages issus du mythe en objets de musée - ces statues vivantes constituant les consommateurs abstraits de leur mythe, ses visiteurs occasionnels, en pleine correspondance avec les touristes contemporains-collectionneurs d'expériences et d'images. Néanmoins, il existe des instants du présent, suggère l'auteur, qui sont peut- 
être gros d'un avenir différent pour le mythe, mais aussi d'une nouvelle consommation de son passé. Les reliefs du festin de Thyeste, qui marquent la mort et la vie qui a été communiée, constituent un seuil, le lieu de rencontre de deux réalités : d'un côté, la désertification du mythe, de l'autre, sa régénération probable.

La désertification du mythe dans le présent s'identifie symboliquement aux reliefs de l'horrible festin offert par Atrée à Thyeste, lorsqu'il lui servit les corps apprêtés de ses enfants : ustensiles et couverts utilisés, petits vêtements d'enfants lacérés et souillés. De l'acte impie du passé mythique ne demeure pour les héroönes de Bruit que le dégoût de ces "reliefs de cent ans" (MATESSIS, 1997, 17) 3 et l'ennuyeuse obligation de la cérémonie en l'honneur de Thyeste au bénéfice de son fils Égisthe. Ainsi, le mythe est demeuré une coquille vide comme le masque d'Agamemnon que porte Électre sous les sarcasmes de Clytemnestre: "Princesse, mon enfant, encore les classiques illustrés ! Quelle monotonie ma chère! Quelle obsession vraiment avec la photographie de papa!» (MATESSIS, 1997, 25)4, ou comme Égisthe qui, cloué dans son fauteuil d'infirme, pousse des cris d'oiseau inarticulés. Clytemnestre, la plaie causée par le poignard meurtrier apparente dans le dos, attend qu'Oreste la tue de nouveau. Les personnages de la tragédie antique ont donc perdu leur élément tragique : il ne reste de leur acte que l'enveloppe extérieure, le geste, coupé de ce qui l'a motivé. Les héros de Bruit vivent l'enfer de la répétition éternelle d'actes qui, soit sont privés de sens, soit sont revêtus de sens étrangers, tels que ceux du christianisme. C'est ainsi qu'Oreste déclare sur un ton déçu: «À présent, comment pourrais-je faire de nous une tragédie? On ne peut guère qu'arriver à Dostoïevski. La mère et le fils, souillés de culpabilités, de remords" (MATESSIS, 1997, 87)5. Pourtant, c'est exactement dans ce mouvement constamment suspendu des personnages que va naître la probabilité de la rupture.

C'est que les reliefs du festin de Thyeste n'appartiennent pas complètement au présent, ils portent encore en eux le poids du passé mythique que la cérémonie commémorative va mettre subitement en lumière. Sur le seuil que forment le lieu et le temps de la cérémonie, la réalité de l'acte anthropophagique revient, intacte, appelant les protagonistes à recueillir leur propre part de cet horrible festin, qui est l'accomplissement douloureux de leur destin. Ainsi, sur le seuil a lieu la rencontre du «terrible», à savoir du sublime, ainsi que le dirait P. Matessis, et du monde désenchanté d'aujourd'hui. De la cérémonie naît aussi le rêve d'Égisthe qui va entraîner Électre et Clytemnestre : «Voilà que maintenant on a attrapé un rêve » (MATESSIS, 1997, $45)^{6}$, dira cette dernière. Puis, dans le rêve est mise en abyme la représentation théâtrale que donne une troupe d'acteurs qui déboule dans le palais. Dans cette représentation, intitulée "La guerre de Troie", les comédiens demandent aux personnages qui rêvent de jouer eux aussi un rôle. Ainsi, hors du rêve, les âges et les qualités des trois personnages - mère, fille, amant - sont fluctuants. Mais la représentation théâtrale mise en abyme dans le rêve, qui constitue un théâtre dans le théâtre pour les spectateurs de Bruit et un rêve dans le rêve pour ses protagonistes, met en lumière des parcelles compactes de l'ethos des personnages.

7 Si, par l'intermédiaire du rêve, les protagonistes retournent au mythe, par le biais du théâtre, ils reviennent à la tragédie antique. Mais dans cette dernière - et c'est là un commentaire ironique de P. Matessis sur la perception du personnage tragique de l'antiquité par le spectateur contemporain - ils ne trouvent pas d'identité propre à leur existence. Ils y reviennent donc en tant qu'acteurs. Ils jouent des rôles à travers lesquels ils peuvent retrouver de petits fragments de leur propre ethos. Mais en 
interprétant un autre rôle que le leur, ils comprennent qu'ils n'étaient eux aussi qu'un rôle. Ils se retrouvent par conséquent face à face avec l'altérité, qui prend deux formes simultanées: celle du passé mythique et celle du présent historique. Même s'ils se reconnaissent tels qu'ils furent à l'intérieur du mythe, ils ne se reconnaissent que comme rôles. Par exemple, en jouant Andromaque, Clytemnestre va se souvenir de sa propre fille Iphigénie et de sa qualité de mère. « Astyanax ! Hector ! Iphigénie [comme si elle s'éveillait] Iphigénie! Iphigénie! Oreste!» (MATESsIs, 1997, 73)7. Au même instant pourtant elle sait que cette qualité n'est rien d'autre qu'une robe sur laquelle est collée l'image d'un petit enfant, et que ce rôle est joué dans un musée sous les directives de comédiens. Ainsi, tandis que les personnages du drame antique semblent retrouver leur identité par la médiation des rôles qu'ils jouent, au même instant ils s'éloignent d'elle, car ils suivent en tant que spectateurs la déliquescence de leur mythe dans le présent: les piètres comédiens représentent le bassin méditerranéen au moyen d'une bassine ${ }^{8}$, ils qualifient d'invités les locataires du palais et les menacent d'interrompre leur rêve s'ils ne suivent pas leurs indications, à savoir de perdre définitivement leur lien avec le mythe.

8 Les métamorphoses que subissent les personnages à l'intérieur du rêve d'Égisthe génèrent deux transformations fondamentales: d'une part, les personnages peuvent être redéfinis comme tragiques, mais il manque à ce tragique actuel l'ombre du destin ou des dieux. À présent, le tragique prend la forme de la distance, du vide, de l'identité délabrée : les héros vivent le passé comme un présent absent, comme un rôle, c'est-àdire comme une illusion. D'autre part, la rupture du temps historique qui s'accomplit par la cérémonie et le rêve permet de nouveau la perception du passé comme interruption dans la continuité et comme novation, ce qui permet donc à l'avenir de dévier de son devenir fixé d'avance, qui est la nécrose du mythe. Dans cette conception du temps conforme à celle envisagée par Walter Benjamin, où le passé est un présent naguère gros de la dynamique du renversement, ou bien d'un présent à l'avenir différent $(1992,254)$, le mythe se défait non seulement des chaînes qui rendaient inactif son présent actif, mais aussi de son passé même. Électre assassine Oreste, et cette version qui diverge du modèle antique souligne le nouveau cycle du sang - Électre, par le meurtre, se place à la tête de la maison des Atrides, devient la nouvelle Clytemnestre. En introduisant le mythe de la Mère-Fille des mystères d'Éleusis, auxquels se réfère d'ailleurs l'un des comédiens tout de suite après le meurtre d'Oreste, P. Matessis met en relief la dynamique du mythe, sa capacité incessante de recomposition. Les reliefs du festin de Thyeste au centre de la scène constituent le rappel constant de cette particularité du mythe.

\section{Le Vertige des animaux avant l'abattage de Dimitris Dimitriadis}

Dans le Vertige des animaux avant l'abattage de D. Dimitriadis est projetée la composition d'une version moderne de Job rehaussée de motifs tragiques antiques: l'ascension sociale fulgurante suivie de la chute et de la destruction d'une famille, découlant d'une prophétie prononcée vingt années auparavant. Philon avait tenté alors de détourner son ami Nilos d'épouser Militssa en lui exposant l'avenir funeste qui l'attendait lui et sa famille: folie, prostitution, inceste, suicide, meurtres. Trois mystérieux personnages désignés par les capitales $\mathrm{A}, \mathrm{B}, \mathrm{C}$ annoncent et commentent l'action. 
10 La langue se trouve au cœur de l'œuvre de D. Dimitriadis qui examine la façon dont la parole se transforme en piège mortel. Le passage à cette fonction dangereuse de la langue s'effectue au cours d'un repas, qui agit comme seuil permettant la coexistence dangereuse de la langue et de l'acte de manger ainsi que leur influence réciproque. Les résultats sont multiples: non seulement le discours prophétique du passé est activé dans le présent par cet acte dès la scène suivante, mais en outre, désormais, la langue entière des personnages est ouverte, suspendue entre la vérité et le mensonge, construisant parfois une relation arbitraire avec l'acte ainsi que le montrent les paroles de Nilos :

[...] mais je ne comprends pas - certaines choses arrivent parce qu'on les a dites et d'autres n'arrivent pas même si on les a dites - les mots n'écoutent pas - ils n'obéissent pas - ils font ce qu'ils veulent - toutes les choses font ce qu'elles veulent.

(DIMITRIADIS, 2008, 130-131)

11 Les personnages rencontrent l'altérité qui prend la forme d'un désir violent pour l'interdit et la destruction - l'altérité provient donc de l'intérieur. Bien sûr, cette métamorphose singulière du discours n'aurait pas eu lieu dans le présent dramatique si la sentence prophétique de Philon n'avait pas précédé. Dans l'une des premières scènes de l'œuvre, parmi les habituelles phrases déclaratives du discours quotidien des deux amis, la prophétie de la destruction imminente surgit sous forme d'enthousiasme au sens propre. Sa connexité avec tout ce qui va se dérouler au cours du repas est claire, puisque la première conjonction entre discours et action survient à ce moment-là. La parole prophétique est une parole performative qui, même si elle s'accomplit vingt ans plus tard, équivaut à une action. Et ce, car dès l'instant où elle est proférée, le champ d'action de l'auditeur est déjà limité : soit il accepte la prophétie, soit il s'éloigne d'elle. Ici, le rôle de la mémoire est crucial, car, quel que soit le chemin suivi par le récepteur de la parole, il sait que ce qui a été dit est peut-être en puissance déjà en cours de réalisation, et que dans un sens ce qui a été dit est déjà advenu, mais aussi continue d'advenir. Ainsi, lorsque Nilos convient qu'il n'a jamais oublié les paroles étranges de son ami et lui demande avec insistance de les répéter, il ne revient pas à l'acte passé comme accompli, mais au passé constamment de retour en tant que présent qui est momentanément absent.

12 Le repas que donne la famille de Philon constitue l'anniversaire des vingt années de mariage du couple, ce qui signifie qu'il s'agit d'un jour tourné vers le passé, d'une journée consacrée au souvenir. C'est précisément dans le cadre du «rituel » qu'est l'anniversaire du mariage, que le souvenir des paroles de Philon va être tiré de l'oubli. D'ailleurs, dès les préparatifs du repas de fête, certains éléments témoignent de la rencontre avec l'altérité toute proche : "C'est comme si quelqu'un nous surveillait", dit Eugénios, l'un des deux fils, «[...] comme s'il dirige ce que nous devons dire, ce que nous devons faire » (DIMITRIADIS, 2008, 43) ${ }^{10}$. Et la mère, un peu plus tard : « Ah, calmezvous un peu. J'ai bien besoin d'un peu de calme, on parle, on parle, et qu'est-ce qu'on dit ? On ne sait même pas. Toutes ces paroles ont l'air d'une maladie » (DIMITRIADIS, 2008, 44) ${ }^{11}$. Le repas de fête débute avec l'arrivée de Philon. Il comprend deux caractéristiques: d'une part, la faim de Starlet, la fille de la maison, ne cesse de s'exprimer, et d'autre part le désir insistant de Nilos qui presse Philon de se souvenir des paroles exactes de l'«oracle». Ce qui débute comme un désir paradoxal de nourriture se poursuit comme un désir également paradoxal d'entendre, de « consommer » les paroles de Philon. Ainsi, ce repas commémoratif dessine un seuil où 
les éléments hétérogènes qui coexistent peuvent se relier de manière imprévue : en effet, les lois qui régissent le repas, le manger et le boire, sont à présent appliquées au discours humain - ainsi, dans un sens, la faim de nourriture se transforme en faim de mots interdits. Ces mots ne sont pas répétés, mais les sous-entendus et les phrases énigmatiques des deux amis amplifient le désir de les entendre, et par conséquent le sentiment géminé de la faim que nous avons mentionné. D'autre part, ils font de ces paroles une forme de discours sacré, de texte inaccessible, selon Philon :

[...] - c'est tellement vieux tout ça - comme si ça sort d'antiques livres - écrits dans une langue inconnue - que même celui qui les a écrits ne sait plus - je me sens si vieux, si ancien. (DIMITRIADIS, 2008, 53) ${ }^{12}$

Sur le plan du symbole, nous pourrions parler d'une incarnation de la parole dans un double sens: la parole, allusive et absente, mais présente à travers le désir des personnages de l'entendre, fonctionne comme chair de la nourriture que consomment - à leur insu? - les convives, et au même instant la parole prophétique du passé, jusqu'à présent soumise au régime de l'acte en puissance, est activée, car elle est désormais une part du corps des personnages.

Tout ce qui va suivre le repas confirme la prophétie. La parole à présent se meut sur un mode arbitraire : tantôt elle correspond aux actes, tantôt elle tente de les prévenir. Le désir de l'autre, qui est en fait le désir de soi, quand l'un des membres de la famille se tourne avec des intentions sexuelles vers un autre, met les personnages face à l'altérité; ainsi Nilos (à sa fille) : «Je ne te veux pas - c'est toi que je veux », « Ne me laisse pas - laisse-moi - Plutôt mourir » (DIMITRIADIs, 2008, 130) ${ }^{13}$. C'est ici l'altérité du même, car les personnages entendent les passions qu'ils vivent à la fois comme le résultat de l'oracle et comme leur propre création. L'épilogue, dans cet univers tragique, sera donné par Philon qui, dans un geste œdipien, se coupera la langue avec des ciseaux.

\section{Le seuil : espace d'apparition de la personne?}

En conclusion, le repas, dans les deux pièces que nous avons étudiées, constitue le champ de rencontre avec le différent. Il introduit l'étranger dans le familier, il fissure le lieu et le temps, il permet l'émergence de l'altérité. Les reliefs du festin de Thyeste mettent les personnages de Bruit face à la nouvelle activation du mythe et à leur rôle dans celui-ci, tandis que le repas de fête des héros de D. Dimitriadis lève soudain les frontières qui séparent le manger et le dire. Pourtant, à l'intérieur du seuil ne vivent pas seulement les personnages dramatiques mais aussi le lecteur/spectateur critique : c'est précisément dans les champs de rupture de la continuité narrative, là où selon Bertolt Brecht $(1974,105)$, les points d'enchaînement des péripéties sont visibles, que peut s'intercaler la dimension critique potentielle du spectateur. Nous pouvons par conséquent supposer que dans l'espace du seuil, auprès du personnage dramatique, s'introduit la notion de la personne humaine selon laquelle l'existence est entendue non comme individualité, mais comme relation, comme orientation et ouverture vers le différent: si la personne n'est pas, dans le sens d'une quelconque essence ou nature, mais se fait, telle une réponse à l'appel de l'altérité, alors le seuil constitue indubitablement l'un des champs où il apparaît. 


\section{BIBLIOGRAPHIE}

BENJAMIN Walter, 1992, Illuminations, Londres : Fontana Press, 1992, cité par STAVRIDIS Stavros

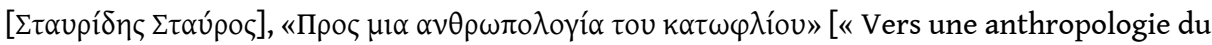
seuil »], Stachy : Outopia, 33, janvier-février 1999, (p. 107-121), p. 116.

BENJAMIN Walter, 2000, Euvres, t. III, Paris : Gallimard.

BOURDIEU Pierre, 2000, Esquisse d'une théorie de la pratique. Précédé de trois études d'ethnologie kabyle, Paris : Seuil, coll. «Point Essais ».

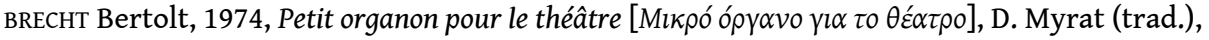
Athènes : Pleias.

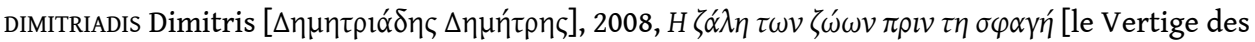
animaux avant l'abattage], Athènes : Indictos.

DIMITRIADIS Dimitris [ $\left.\Delta \eta \mu \eta \tau \rho{ }^{\alpha} \delta \eta \varsigma \Delta \eta \mu \eta ́ \tau \rho \eta \varsigma\right], 2009$, le Vertige des animaux avant l'abattage, Besançon : Les Solitaires intempestifs, trad. Olivier Goetz et Armando Llamas.

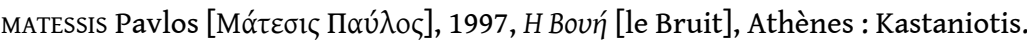

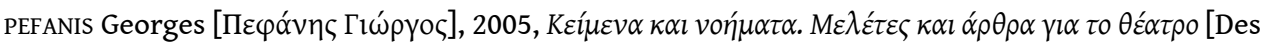
textes et des sens. Études et articles sur le théâtre], Athènes : Sokolis.

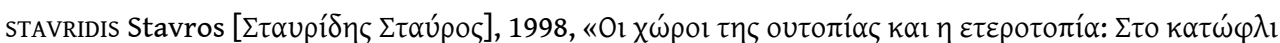

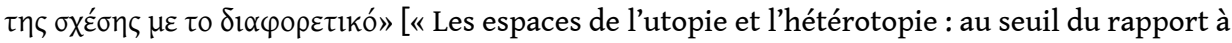
l'autre »], Stachy : Outopia, 31, septembre-octobre 1998, p. 51-66.

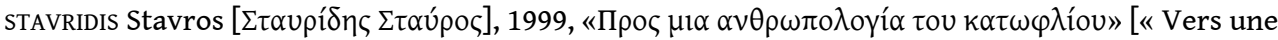
anthropologie du seuil »], Stachy : Outopia, 33, janvier-février 1999, p. 107-121.

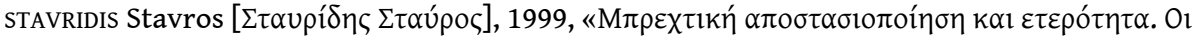

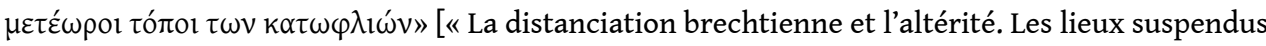
des seuils »], Synchrona themata, 71-72, avril-septembre 1999, p. 94-98.

\section{NOTES}

\section{H Bouń.}

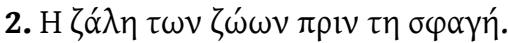

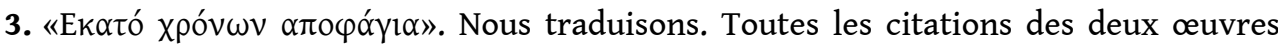
dramatiques sont dues à nos soins.

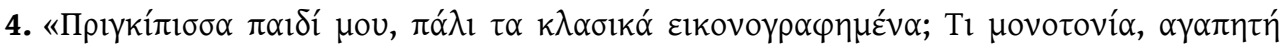

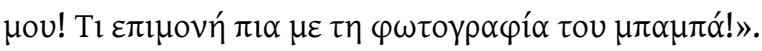

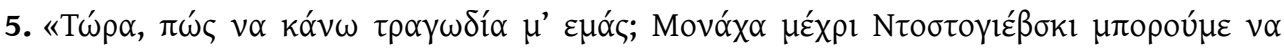

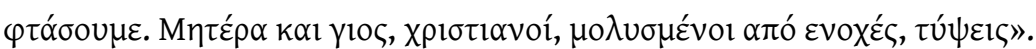

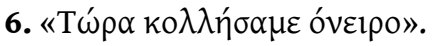

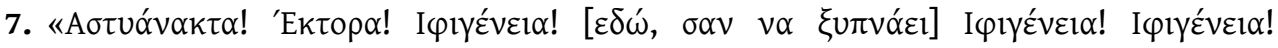

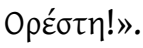


8. "Amer commentaire sur les dimensions désormais perdues de la grandeur de

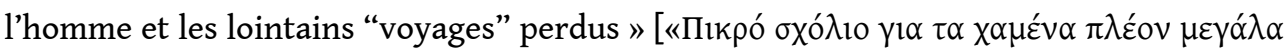

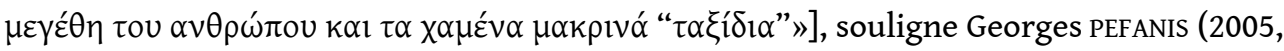
148).

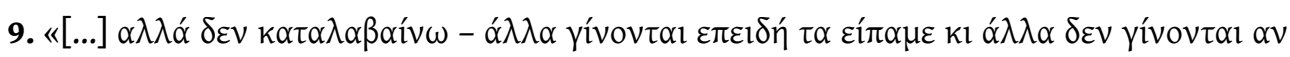

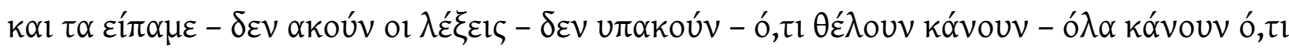
$\theta \varepsilon ́ \lambda o u v »$.

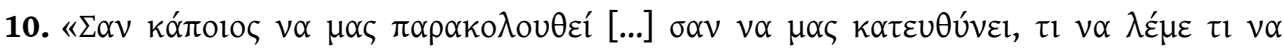

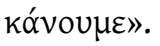

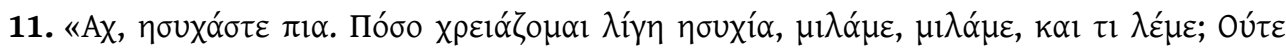

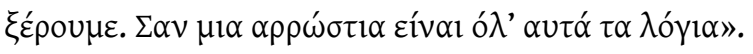

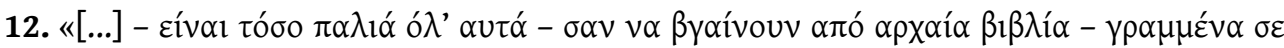

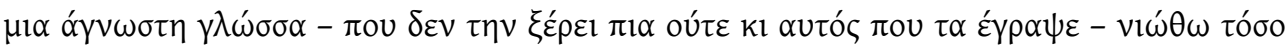

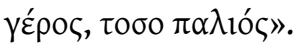

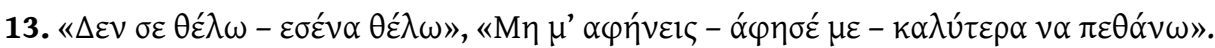

\section{RÉSUMÉS}

Le repas dans les pièces le Bruit de Pavlos Matessis et le Vertige des animaux avant l'abattage de Dimitris Dimitriadis constitue un seuil d'où émerge l'altérité. Dans le Bruit, le repas réactive le mythe ancien; dans la pièce de Dimitriadis, il lève soudain les frontières qui séparent le manger et le dire, produisant ainsi un mélange dangereux.

In plays such as P. Matesis's The sound and D. Dimitriadis The vertigo of animals before the slaughter, the act of eating forms a threshold opened to alterity. In the first case, dinner provokes the reactivation of the ancient myth. In the second case, it brings to light the dangerous commixture of flesh and speech.

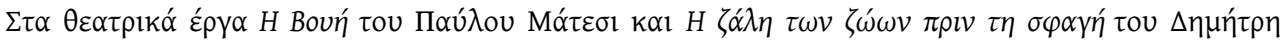

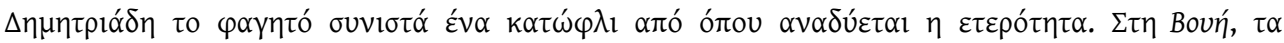

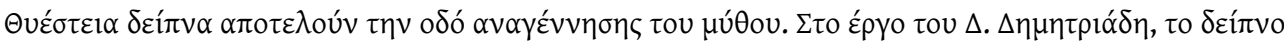

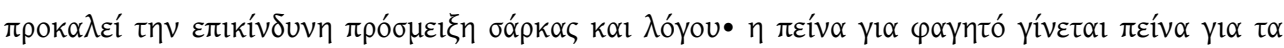

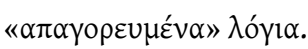


INDEX

Index géographique : Grèce

motsclestr Yemek, Eşik, Ötekilik, Kimlik, Efsane, Yunanistan, Yirminci yüzyıl, Tiyatro

Keywords : Dinner, Threshold, Alterity, Identity, Myth, Greece, Twentieth Century, Theatre, Tragique

Mots-clés : repas, repas, seuil, seuil, altérité, altérité, identité, identité, mythe, mythe, Dimitriadis Dimitris (1944-), Dimitriadis Dimitris (1944-), Matessis Pavlos (1933-2013), Matessis Pavlos (1933-2013)

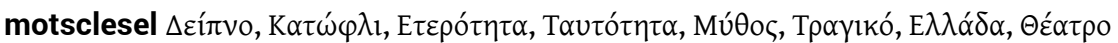

Thèmes : Théâtre

motsclesmk ОБРОЦИ, ПРАГ, ДРУГОСТА, ИДЕНТИТЕТ, МИТ, ГРЦИЈА, ДВАЕСЕТТИОТ ВЕК, ТЕАТАР

Index chronologique : vingtième siècle

\section{AUTEUR}

\section{THALIA BOUSIOPOULOU}

Université de Lille 